\title{
Some Weighted Aggregation Operators of Trapezoidal Neutrosophic Numbers and Their Multiple Attribute Decision Making Method
}

\author{
Jun YE \\ Department of Electrical and Information Engineering, Shaoxing University \\ 508 Huancheng West Road, Shaoxing, Zhejiang Province 312000, PR China \\ e-mail:yehjun@aliyun.com
}

Received: January 2015; accepted: January 2016

\begin{abstract}
This paper proposes the concepts of a neutrosophic number and a trapezoidal neutrosophic number (TNN), the basic operational relations of TNNs, and the score function of TNN. Then, we develop a trapezoidal neutrosophic weighted arithmetic averaging (TNWAA) operator and a trapezoidal neutrosophic weighted geometric averaging (TNWGA) operator to aggregate TNN information and investigate their properties. Furthermore, a multiple attribute decision making method based on the TNWAA and TNWGA operators and the score function of TNN is established under a TNN environment. Finally, an illustrative example of investment alternatives is given to demonstrate the application and effectiveness of the developed approach.
\end{abstract}

Key words: trapezoidal neutrosophic number, score function, trapezoidal neutrosophic weighted arithmetic averaging (TNWAA) operator, trapezoidal neutrosophic weighted geometric averaging (TNWGA) operator, multiple attribute decision making.

\section{Introduction}

Fuzzy decision making is an important topic in decision theory. Recently, many researchers have proposed various fuzzy decision making methods (Liu and Yu, 2013; Meng and Chen, 2014; Wang and Liu, 2014; Zhou and He, 2014; Wan and Dong, 2014). However, these methods cannot handle decision-making problems with indeterminate and inconsistent information. Then, Smarandache (1999) originally gave a concept of a neutrosophic set, which is a part of neutrosophy and generalizes fuzzy sets (Zadeh, 1965), interval valued fuzzy sets (IVFSs) (Turksen, 1986), intuitionistic fuzzy sets (IFSs) (Atanassov, 1986), and interval-valued intuitionistic fuzzy sets (IVIFSs) (Atanassov and Gargov, 1989) from philosophical point of view. To obtain the real applications, Wang et al. $(2005,2010)$ presented single valued neutrosophic sets (SVNSs) and interval neutrosophic sets (INSs), which are the subclasses of neutrosophic sets. They can independently express the truthmembership degree, indeterminacy-membership degree and false-membership degree. SVNSs and INSs, as the generalization of IFSs and IVIFSs, can handle incomplete, indeterminate and inconsistent information which exists commonly in real situations, while 
IFSs and IVIFSs only express truth-membership degree and false-membership degree, but cannot deal with indeterminate and inconsistent information. Hence, SVNSs and INSs are very suitable for applications in decision making. Ye (2013) developed the correlation coefficient of SVNSs as the extension of the correlation coefficient of IFSs and proved that the cosine similarity measure of SVNSs is a special case of the correlation coefficient of SVNSs, and then applied it to single valued neutrosophic decision-making problems. Chi and Liu (2013) proposed an extended TOPSIS method for multiple attribute decision making under an interval neutrosophic environment. Moreover, Ye (2014a) presented the Hamming and Euclidean distances between INSs and the distances-based similarity measures of INSs, and then a multi criteria decision making method based on the similarity measures of INSs was established in interval neutrosophic setting. Furthermore, Ye (2014b) proposed a cross-entropy measure of SVNSs and applied it to multi criteria decision making problems with single valued neutrosophic information. Ye (2014c) further introduced a simplified neutrosophic set (SNS) as a subclass of a neutrosophic set, which includes SVNS and INS, and developed a simplified neutrosophic weighted averaging (SNWA) operator and a simplified neutrosophic weighted geometric (SNWG) operator, and then he applied them to multi criteria decision making under a simplified neutrosophic environment. Liu et al. (2014) further proposed some generalized single valued neutrosophic number Hamacher aggregation operators and applied them to group decision making. Then, Zhang et al. (2014) defined the score, accuracy and certainty functions for interval neutrosophic numbers (INNs) and presented a comparative approach for INNs, and then they also developed some aggregation operators for INNs and a multi criteria decision-making method by means of the aggregation operators. On the other hand, Ye (2014d) put forward vector similarity measures, including the Dice, Jaccard and cosine measures of SNSs, and applied them to multi criteria decision-making problems in simplified neutrosophic setting. Biswas et al. (2014) established a single valued neutrosophic multiple attribute decision making method with unknown weight information, where optimization models were used to determine unknown attribute weights and the grey relational coefficient of each alternative from ideal alternative was utilized to rank alternatives. Zhang and $\mathrm{Wu}$ (2014) also developed a method for solving single valued neutrosophic multi criteria decision making problems with incomplete weight information, in which the criterion values are given in the form of single-valued neutrosophic sets (SVNSs), and the information about criterion weights is incompletely known or completely unknown. Also, Broumi and Smarandache $(2014,2015)$ further presented the cosine similarity measure and new operations of INNs. Ye (2014e) proposed the weighted arithmetic average and weighted geometric average operators of interval neutrosophic linguistic numbers (INLNs) and applied them to multiple attribute decision making problems with interval neutrosophic linguistic information.

Intuitionistic fuzzy numbers (IFNs) and intuitionistic trapezoidal fuzzy numbers (ITFNs) introduced in Wang and Zhang (2009) are the extension of IFSs in another way, which extend discrete sets to continuous sets. Then the domains of SVNSs and INSs are discrete sets, but not continuous sets in existing literature. The advantage of continuous sets is that they include much information and the fuzziness in multiple attribute decision making has the better character because of the proposal of fuzzy number (Wang and 
Zhang, 2009). Liu and Yu (2013) proposed density aggregation operators of ITFNs for multiple attribute decision making. Wan and Dong (2014) developed a multi-attribute group decision making method with trapezoidal intuitionistic fuzzy numbers and applied it to stock selection.

Furthermore, the domains of SVNSs and INSs are discrete sets, but not continuous sets in existing literature. At present, there are no studies on neutrosophic numbers and trapezoidal neutrosophic numbers (TNNs) in above mentioned decision-making problems. Motivated by Wang and Zhang (2009), we should make the truth-membership, indeterminacymembership and falsity-membership degrees in an SVNS or an INS no longer relative to single or interval values, but relative to fuzzy numbers or trapezoidal fuzzy numbers. Thus we can introduce the concepts of neutrosophic numbers and TNNs to extend the discrete domains of SVNSs and INSs to continuous domains of TNNs, which are also the further extension of IFNs and ITFNs (Wang and Zhang, 2009). However, a TNN is a special case of a neutrosophic number and useful in practical applications. Then, the typical TNN is of importance for neutrosophic multiple attribute decision making problems. Therefore, the purposes of this article are: (1) to introduce the concepts of a neutrosophic number and a TNN, some basic operational relations of TNNs and a score function for a TNN, (2) to propose two aggregation operators: a trapezoidal neutrosophic weighted arithmetic averaging (TNWAA) operator and a trapezoidal neutrosophic weighted geometric averaging (TNWGA) operator, and (3) to establish a decision making approach based on the TNWAA and TNWGA operators and the score function under a TNN environment.

The rest of the article is organized as follows. Section 2 briefly describes some concepts of IFNs, ITFNs and operational relations for ITFNs. Section 3 proposes the concepts of a neutrosophic number and a TNN and defines some basic operations of TNNs and the score function of a TNN. In Section 4, we develop TNWAA and TNWGA operators for TNNs and investigate their properties. Section 5 establishes a decision making approach based on the TNWAA and TNWGA operators and the score function under a TNN environment. In Section 6, an illustrative example is provided to illustrate the application of the developed method. Section 7 contains conclusions and future research.

\section{Intuitionistic Fuzzy Numbers and Intuitionistic Trapezoidal Fuzzy Numbers}

In this section, we briefly describe some concepts of IFNs, ITFNs and operational relations for ITFNs.

Definition 1. (See Wang and Zhang, 2009.) Let $\tilde{a}$ be an IFN in the set of real numbers $R$, then its membership function is defined as

$$
\mu_{\tilde{a}}(x)= \begin{cases}f_{\tilde{a}}(x), & a_{1} \leqslant x<a_{2}, \\ \mu_{\tilde{a}}, & a_{2} \leqslant x \leqslant a_{3}, \\ g_{\tilde{a}}(x), & a_{3}<x \leqslant a_{4}, \\ 0, & \text { otherwise }\end{cases}
$$


and its nonmembership function is defined as

$$
v_{\tilde{a}}(x)= \begin{cases}h_{\tilde{a}}(x), & b_{1} \leqslant x<b_{2}, \\ v_{\tilde{a}}, & b_{2} \leqslant x \leqslant b_{3}, \\ k_{\tilde{a}}(x), & b_{3}<x \leqslant b_{4}, \\ 1, & \text { otherwise }\end{cases}
$$

where $\mu_{\tilde{a}}, v_{\tilde{a}} \in[0,1], 0 \leqslant \mu_{\tilde{a}}+v_{\tilde{a}} \leqslant 1$ and $a_{1}, a_{2}, a_{3}, a_{4}, b_{1}, b_{2}, b_{3}, b_{4} \in R$, and four functions $f_{\tilde{a}}, g_{\tilde{a}}, h_{\tilde{a}}, k_{\tilde{a}}: R \rightarrow[0,1]$ are called the side of a fuzzy number. The functions $f_{\tilde{a}}$ and $k_{\tilde{a}}$ are increasing continuous functions, and then the functions $g_{\tilde{a}}$ and $h_{\tilde{a}}$ are decreasing continuous functions.

Particularly, if the increasing functions $f_{\tilde{a}}$ and $k_{\tilde{a}}$ and decreasing functions $g_{\tilde{a}}$ and $h_{\tilde{a}}$ are linear, then we have ITFNs, which are preferred in practice.

Definition 2. (See Wang and Zhang, 2009.) Let $\tilde{a}$ be an ITFN. Then, the membership function and nonmembership function can be defined, respectively, as follows:

$$
\begin{aligned}
& \mu_{\tilde{a}}(x)= \begin{cases}\frac{x-a_{1}}{a_{2}-a_{1}} \mu_{\tilde{a}}, & a_{1} \leqslant x<a_{2}, \\
\mu_{\tilde{a}}, & a_{2} \leqslant x \leqslant a_{3}, \\
\frac{a_{4}-x}{a_{4}-a_{3}} \mu_{\tilde{a}}, & a_{3}<x \leqslant a_{4}, \\
0, & \text { otherwise, }\end{cases} \\
& v_{\tilde{a}}(x)= \begin{cases}\frac{b_{2}-x+v_{\tilde{a}}\left(x-b_{1}\right)}{b_{2}-b_{1}}, & b_{1} \leqslant x<b_{2}, \\
\frac{v_{\tilde{a}},}{x-b_{3}+v_{\tilde{a}}\left(b_{4}-x\right)} & b_{2} \leqslant x \leqslant b_{3}, \\
b_{4}-b_{3}, & a_{3}<x \leqslant a_{4}, \\
1, & \text { otherwise }\end{cases}
\end{aligned}
$$

where $\mu_{\tilde{a}}, v_{\tilde{a}} \in[0,1], 0 \leqslant \mu_{\tilde{a}}+v_{\tilde{a}} \leqslant 1$ and $a_{1}, a_{2}, a_{3}, a_{4}, b_{1}, b_{2}, b_{3}, b_{4} \in R$. Generally, if $\left[a_{1}, a_{2}, a_{3}, a_{4}\right]=\left[b_{1}, b_{2}, b_{3}, b_{4}\right]$ in an ITFN $\tilde{a}$, then the ITFN $\tilde{a}$ is denoted as $\tilde{a}=\left\langle\left(a_{1}, a_{2}, a_{3}, a_{4}\right) ; \mu_{\tilde{a}}, v_{\tilde{a}}\right\rangle$.

ITFNs have the following operational relations (Wang and Zhang, 2009).

Definition 3. (See Wang and Zhang, 2009.) Let $\tilde{a}=\left\langle\left(a_{1}, a_{2}, a_{3}, a_{4}\right) ; \mu_{\tilde{a}}, \nu_{\tilde{a}}\right\rangle$ and $\tilde{b}=$ $\left\langle\left(b_{1}, b_{2}, b_{3}, b_{4}\right) ; \mu_{\tilde{b}}, v_{\tilde{b}}\right\rangle$ be two ITFNs and $\lambda \geqslant 0$. Then there are the following operational relations:

(1) $\tilde{a}+\tilde{b}=\left\langle\left(a_{1}+b_{1}, a_{2}+b_{2}, a_{3}+b_{3}, a_{4}+b_{4}\right) ; \mu_{\tilde{a}}+\mu_{\tilde{b}}-\mu_{\tilde{a}} \mu_{\tilde{b}}, v_{\tilde{a}} v_{\tilde{b}}\right\rangle$;

(2) $\tilde{a} \tilde{b}=\left\langle\left(a_{1} b_{1}, a_{2} b_{2}, a_{3} b_{3}, a_{4} b_{4}\right) ; \mu_{\tilde{a}} \mu_{\tilde{b}}, v_{\tilde{a}}+v_{\tilde{b}}-v_{\tilde{a}} v_{\tilde{b}}\right\rangle$;

(3) $\lambda \tilde{a}=\left\langle\left(\lambda a_{1}, \lambda a_{2}, \lambda a_{3}, \lambda a_{4}\right) ; 1-\left(1-\mu_{\tilde{a}}\right)^{\lambda}, \nu_{\tilde{a}}^{\lambda}\right\rangle$;

(4) $\tilde{a}^{\lambda}=\left\langle\left(a_{1}^{\lambda}, a_{2}^{\lambda}, a_{3}^{\lambda}, a_{4}^{\lambda}\right) ; \mu_{\tilde{a}}^{\lambda}, 1-\left(1-v_{\tilde{a}}\right)^{\lambda}\right\rangle$. 


\section{Neutrosophic Numbers and Trapezoidal Neutrosophic Numbers}

In this section, motivated by IFNs and ITFNs, we propose neutrosophic numbers and TNNs based on the combination of SVNSs and fuzzy numbers as the generalization of IFNs and ITFNs, which extend discrete sets to continuous sets. Smarandache (1999) firstly presented a neutrosophic set from philosophical point of view. To easily apply the neutrosophic set to practical problems, Wang et al. (2010) introduced the concept of an SVNS, which is a subclass of the neutrosophic set.

Definition 4. (See Wang et al., 2010.) Let $X$ be a space of points (objects) with generic elements in $X$ denoted by $x$. An SVNS $N$ in $X$ is characterized by a truth-membership function $T_{N}(x)$, an indeterminacy-membership function $I_{N}(x)$ and a falsity-membership function $F_{N}(x)$. Then, an SVNS $N$ can be denoted by

$$
N=\left\{\left\langle x, T_{N}(x), I_{N}(x), F_{N}(x)\right\rangle \mid x \in X\right\},
$$

where the sum of $T_{N}(x), I_{N}(x), F_{N}(x) \in[0,1]$ satisfies $0 \leqslant T_{N}(x)+I_{N}(x)+F_{N}(x) \leqslant 3$ for each point $x$ in $X$. For convenience, we can use the simplified symbol $n_{x}=\left\langle T_{x}, I_{x}, F_{x}\right\rangle$ to represent a basic element in an SVNS $N$, and call it a single valued neutrosophic number (SVNN).

Different from the definition of an SVNS, we make the truth-membership, indeterminacymembership and falsity-membership degrees no longer relative to single values, but relative to fuzzy numbers. Then, we can give the following definitions of a neutrosophic number and a TNN.

Definition 5. Let $\tilde{N}$ be a neutrosophic number in the set of real numbers $R$, then its truth-membership function is defined as

$$
T_{\tilde{n}}(x)= \begin{cases}f_{\tilde{n}}(x), & a_{1} \leqslant x<a_{2}, \\ T_{\tilde{n}}, & a_{2} \leqslant x \leqslant a_{3}, \\ g_{\tilde{n}}(x), & a_{3}<x \leqslant a_{4}, \\ 0, & \text { otherwise }\end{cases}
$$

its indeterminacy-membership function is defined as

$$
I_{\tilde{n}}(x)= \begin{cases}h_{\tilde{n}}(x), & b_{1} \leqslant x<b_{2} \\ I_{\tilde{n}}, & b_{2} \leqslant x \leqslant b_{3} \\ k_{\tilde{n}}(x), & b_{3}<x \leqslant b_{4} \\ 1, & \text { otherwise }\end{cases}
$$


and its falsity-membership function is defined as

$$
F_{\tilde{n}}(x)= \begin{cases}p_{\tilde{n}}(x), & c_{1} \leqslant x<c_{2}, \\ F_{\tilde{n}}, & c_{2} \leqslant x \leqslant c_{3}, \\ q_{\tilde{n}}(x), & c_{3}<x \leqslant c_{4}, \\ 1, & \text { otherwise }\end{cases}
$$

where $T_{\tilde{n}}, I_{\tilde{n}}, F_{\tilde{n}} \in[0,1], 0 \leqslant T_{\tilde{n}}+I_{\tilde{n}}+F_{\tilde{n}} \leqslant 3$ and $a_{1}, a_{2}, a_{3}, a_{4}, b_{1}, b_{2}, b_{3}, b_{4}, c_{1}, c_{2}$, $c_{3}, c_{4} \in R$, and six functions $f_{\tilde{n}}, g_{\tilde{n}}, h_{\tilde{n}}, k_{\tilde{n}}, p_{\tilde{n}}, q_{\tilde{n}}: R \rightarrow[0,1]$ are called the side of a fuzzy number. The functions $f_{\tilde{n}}, k_{\tilde{n}}$ and $q_{\tilde{n}}$ are increasing continuous functions, and then the functions $g_{\tilde{n}}, h_{\tilde{n}}$ and $p_{\tilde{n}}$ are decreasing continuous functions.

Especially, if the increasing functions $f_{\tilde{n}}, k_{\tilde{n}}, q_{\tilde{n}}$ and the decreasing functions $g_{\tilde{n}}, h_{\tilde{n}}, p_{\tilde{n}}$ are linear, then we have a TNN, which is preferred in practice.

Definition 6. Let $\tilde{n}$ be a TNN. Then, the truth-membership function, indeterminacymembership function and falsity-membership function can be defined, respectively, as follows:

$$
\begin{gathered}
T_{\tilde{n}}(x)= \begin{cases}\frac{x-a_{1}}{a_{2}-a_{1}} T_{\tilde{n}}, & a_{1} \leqslant x<a_{2}, \\
T_{\tilde{n}}, & a_{2} \leqslant x \leqslant a_{3}, \\
\frac{a_{4}-x}{a_{4}-a_{3}} T_{\tilde{n}}, & a_{3}<x \leqslant a_{4}, \\
0, & \text { otherwise }\end{cases} \\
I_{\tilde{n}}(x)= \begin{cases}\frac{b_{2}-x+I_{\tilde{n}}\left(x-b_{1}\right)}{b_{2}-b_{1}}, & b_{1} \leqslant x<b_{2}, \\
I_{\tilde{n}}, & b_{2} \leqslant x \leqslant b_{3}, \\
\frac{x-b_{3}+I_{\tilde{n}}\left(b_{4}-x\right)}{b_{4}-b_{3}}, & a_{3}<x \leqslant a_{4}, \\
1, & \text { otherwise }\end{cases} \\
F_{\tilde{n}}(x)= \begin{cases}\frac{c_{2}-x+F_{\tilde{n}}\left(x-c_{1}\right)}{c_{2}-c_{1}}, & c_{1} \leqslant x<c_{2}, \\
\frac{F_{\tilde{n}},}{x-c_{3}+F_{\tilde{n}}\left(c_{4}-x\right)} & c_{2} \leqslant x \leqslant c_{3}, \\
c_{4}-c_{3} & c_{3}<x \leqslant c_{4},\end{cases} \\
1, \quad \text { otherwise }
\end{gathered}
$$

where $T_{\tilde{n}}, I_{\tilde{n}}, F_{\tilde{n}} \in[0,1], 0 \leqslant T_{\tilde{n}}+I_{\tilde{n}}+F_{\tilde{n}} \leqslant 3$ and $a_{1}, a_{2}, a_{3}, a_{4}, b_{1}, b_{2}, b_{3}, b_{4}, c_{1}, c_{2}$, $c_{3}, c_{4} \in R$. Then, $\tilde{n}=\left\langle\left(\left[a_{1}, a_{2}, a_{3}, a_{4}\right] ; T_{\tilde{n}}\right),\left(\left[b_{1}, b_{2}, b_{3}, b_{4}\right] ; I_{\tilde{n}}\right),\left(\left[c_{1}, c_{2}, c_{3}, c_{4}\right] ; F_{\tilde{n}}\right)\right\rangle$ is called a TNN. Generally, if $\left[a_{1}, a_{2}, a_{3}, a_{4}\right]=\left[b_{1}, b_{2}, b_{3}, b_{4}\right]=\left[c_{1}, c_{2}, c_{3}, c_{4}\right]$ in a TNN $\tilde{n}$, then the TNN $\tilde{n}$ can be denoted as $\tilde{n}=\left\langle\left(a_{1}, a_{2}, a_{3}, a_{4}\right) ; T_{\tilde{n}}, I_{\tilde{n}}, F_{\tilde{n}}\right\rangle$. 
If $a_{2}=a_{3}$ in a TNN $\tilde{n}$, the TNN $\tilde{n}$ reduces to the triangular neutrosophic number, which is considered as a special case of the TNN $\tilde{n}$. If $a_{1}=a_{2}=a_{3}=a_{4}=1$ in a TNN $\tilde{n}$, then the TNN $\tilde{n}$ reduces to the SVNN.

If $0 \leqslant a_{1} \leqslant a_{2} \leqslant a_{3} \leqslant a_{4}$, then $\tilde{n}$ is called a positive TNN. If $a_{1} \leqslant a_{2} \leqslant a_{3} \leqslant a_{4} \leqslant 0$, then $\tilde{n}$ is called a negative TNN. If $0 \leqslant a_{1} \leqslant a_{2} \leqslant a_{3} \leqslant a_{4} \leqslant 1$ and $T_{\tilde{n}}, I_{\tilde{n}}, F_{\tilde{n}} \in[0,1]$, then $\tilde{n}$ is called a normalized TNN, which is used for this paper.

Thus, we can introduce the following operational relations of TNNs:

Definition 7. Let $\tilde{n}_{1}=\left\langle\left(a_{1}, a_{2}, a_{3}, a_{4}\right) ; T_{\tilde{n}_{1}}, I_{\tilde{n}_{1}}, F_{\tilde{n}_{1}}\right\rangle$ and $\tilde{n}_{2}=\left\langle\left(b_{1}, b_{2}, b_{3}, b_{4}\right) ; T_{\tilde{n}_{2}}\right.$, $\left.I_{\tilde{n}_{2}}, F_{\tilde{n}_{2}}\right\rangle$ be two TNNs and $\lambda \geqslant 0$. Then there are the following operational relations:

(1) $\tilde{n}_{1}+\tilde{n}_{2}=\left\langle\left(a_{1}+b_{1}, a_{2}+b_{2}, a_{3}+b_{3}, a_{4}+b_{4}\right) ; T_{\tilde{n}_{1}}+T_{\tilde{n}_{2}}-T_{\tilde{n}_{1}} T_{\tilde{n}_{2}}, I_{\tilde{n}_{1}} I_{\tilde{n}_{2}}, F_{\tilde{n}_{1}} F_{\tilde{n}_{2}}\right\rangle$;

(2) $\tilde{n}_{1} \tilde{n}_{2}=\left\langle\left(a_{1} b_{1}, a_{2} b_{2}, a_{3} b_{3}, a_{4} b_{4}\right) ; T_{\tilde{n}_{1}} T_{\tilde{n}_{2}}, I_{\tilde{n}_{1}}+I_{\tilde{n}_{2}}-I_{\tilde{n}_{1}} I_{\tilde{n}_{2}}, F_{\tilde{n}_{1}}+F_{\tilde{n}_{2}}-F_{\tilde{n}_{1}} F_{\tilde{n}_{2}}\right\rangle$;

(3) $\lambda \tilde{n}_{1}=\left\langle\left(\lambda a_{1}, \lambda a_{2}, \lambda a_{3}, \lambda a_{4}\right) ; 1-\left(1-T_{\tilde{n}_{1}}\right)^{\lambda}, I_{\tilde{n}_{1}}^{\lambda}, F_{\tilde{n}_{1}}^{\lambda}\right\rangle$;

(4) $\tilde{n}_{1}^{\lambda}=\left\langle\left(a_{1}^{\lambda}, a_{2}^{\lambda}, a_{3}^{\lambda}, a_{4}^{\lambda}\right) ; T_{\tilde{n}_{1}}^{\lambda}, 1-\left(1-I_{\tilde{n}_{1}}\right)^{\lambda}, 1-\left(1-F_{\tilde{n}_{1}}\right)^{\lambda}\right\rangle$.

Based on the expected value of an ITFN (Wang and Zhang, 2009) and the score function of an interval neutrosophic value (Zhang et al., 2014), we can give the following definition of a score function for a TNN.

Definition 8. Let $\tilde{n}=\left\langle\left(a_{1}, a_{2}, a_{3}, a_{4}\right) ; T_{\tilde{n}}, I_{\tilde{n}}, F_{\tilde{n}}\right\rangle$ be a TNN. Then there is the score function of $\tilde{n}$ :

$$
S(\tilde{n})=\frac{1}{12}\left(a_{1}, a_{2}, a_{3}, a_{4}\right)\left(2+T_{\tilde{n}}-I_{\tilde{n}}-F_{\tilde{n}}\right), \quad S(\tilde{n}) \in[0,1] .
$$

For the comparison between two TNNs, a comparative method based on the score function is defined as follows.

Definition 9. Let $\tilde{n}_{1}=\left\langle\left(a_{1}, a_{2}, a_{3}, a_{4}\right) ; T_{\tilde{n}_{1}}, I_{\tilde{n}_{1}}, F_{\tilde{n}_{1}}\right\rangle$ and $\tilde{n}_{2}=\left\langle\left(b_{1}, b_{2}, b_{3}, b_{4}\right) ; T_{\tilde{n}_{2}}\right.$, $\left.I_{\tilde{n}_{2}}, F_{\tilde{n}_{2}}\right\rangle$ be two TNNs. If $S\left(\tilde{n}_{1}\right)>S\left(\tilde{n}_{2}\right)$, then $\tilde{n}_{1}>\tilde{n}_{2}$; if $S\left(\tilde{n}_{1}\right)=S\left(\tilde{n}_{2}\right)$, then $\tilde{n}_{1}=\tilde{n}_{2}$.

For example, let two TNNs be $\tilde{n}_{1}=\langle(0.4,0.5,0.6,0.7) ; 0.4,0.2,0.3\rangle$ and $\tilde{n}_{2}=$ $\langle(0.6,0.7,0.8,0.9) ; 0.6,0.3,0.4\rangle$. In this case, we can compare them according to the score values. Since $S\left(\tilde{n}_{1}\right)=(0.4+0.5+0.6+0.7)(2+0.4-0.2-0.3) \div 12=0.3483$ and $S\left(\tilde{n}_{2}\right)=(0.6+0.7+0.8+0.9)(2+0.6-0.3-0.4) \div 12=0.475$, by Definition 9 , there is $\tilde{n}_{1}<\tilde{n}_{2}$.

\section{Two Weighted Aggregation Operators of TNNs}

Since aggregation operators are an important tool for aggregated information in decisionmaking process, this section proposes two weighted aggregation operators to aggregate TNNs as a generalization of the weighted aggregation operators for ITFNs (Wang and Zhang, 2009), which are usually used in decision making. 
4.1. Trapezoidal Neutrosophic Weighted Arithmetic Averaging Operator

Definition 10. Let $\tilde{n}_{j}=\left\langle\left(a_{j 1}, a_{j 2}, a_{j 3}, a_{j 4}\right) ; T_{\tilde{n}_{j}}, I_{\tilde{n}_{j}}, F_{\tilde{n}_{j}}\right\rangle(j=1,2, \ldots, n)$ be a collection of TNNs, then a TNWAA operator is defined as follows:

$$
\operatorname{TNWAA}\left(\tilde{n}_{1}, \tilde{n}_{2}, \ldots, \tilde{n}_{n}\right)=\sum_{j=1}^{n} w_{j} \tilde{n}_{j}
$$

where $w_{j}$ is the weight of $\tilde{n}_{j}(j=1,2, \ldots, n)$ such that $w_{j}>0$ and $\sum_{j=1}^{n} w_{j}=1$. Specially, when $w_{j}=1 / n$ for $j=1,2, \ldots, n$, the TNWAA operator reduces to the trapezoidal neutrosophic arithmetic averaging operator.

According to Definitions 7 and 10, we can introduce the following theorem.

Theorem 1. Let $\tilde{n}_{j}=\left\langle\left(a_{j 1}, a_{j 2}, a_{j 3}, a_{j 4}\right) ; T_{\tilde{n}_{j}}, I_{\tilde{n}_{j}}, F_{\tilde{n}_{j}}\right\rangle(j=1,2, \ldots, n)$ be a collection of TNNs, then according to Definitions 7 and 10, we can give the following TNWAA operator:

$$
\begin{aligned}
\operatorname{TNWAA}\left(\tilde{n}_{1}, \tilde{n}_{2}, \ldots, \tilde{n}_{n}\right)= & \sum_{j=1}^{n} w_{j} \tilde{n}_{j} \\
= & \left\langle\left(\sum_{j=1}^{n} w_{j} a_{j 1}, \sum_{j=1}^{n} w_{j} a_{j 2}, \sum_{j=1}^{n} w_{j} a_{j 3}, \sum_{j=1}^{n} w_{j} a_{j 4}\right) ;\right. \\
& \left.1-\prod_{j=1}^{n}\left(1-T_{\tilde{n}_{j}}\right)^{w_{j}}, \prod_{j=1}^{n} I_{\tilde{n}_{j}}^{w_{j}}, \prod_{j=1}^{n} F_{\tilde{n}_{j}}^{w_{j}}\right\rangle
\end{aligned}
$$

where $w_{j}$ is the weight of $\tilde{n}_{j}(j=1,2, \ldots, n)$ such that $w_{j}>0$ and $\sum_{j=1}^{n} w_{j}=1$.

Theorem 1 can be proved by means of mathematical induction.

Proof. (1) When $n=2$, then,

$$
\begin{aligned}
& w_{1} \tilde{n}_{1}=\left\langle\left(w_{1} a_{11}, w_{1} a_{12}, w_{1} a_{13}, w_{1} a_{14}\right) ; 1-\left(1-T_{\tilde{n}_{1}}\right)^{w_{1}}, I_{\tilde{n}_{1}}^{w_{1}}, F_{\tilde{n}_{1}}^{w_{1}}\right\rangle, \\
& w_{2} \tilde{n}_{2}=\left\langle\left(w_{2} a_{21}, w_{2} a_{22}, w_{2} a_{23}, w_{2} a_{24}\right) ; 1-\left(1-T_{\tilde{n}_{2}}\right)^{w_{2}}, I_{\tilde{n}_{2}}^{w_{2}}, F_{\tilde{n}_{2}}^{w_{2}}\right\rangle .
\end{aligned}
$$

Thus,

$$
\begin{aligned}
\operatorname{TNWAA}\left(\tilde{n}_{1}, \tilde{n}_{2}\right)= & w_{1} \tilde{n}_{1}+w_{2} \tilde{n}_{2} \\
= & \left\langle\left( w_{1} a_{11}+w_{2} a_{21}, w_{1} a_{12}+w_{2} a_{22},\right.\right. \\
& \left.w_{1} a_{13}+w_{2} a_{23}, w_{1} a_{14}+w_{2} a_{24}\right) ;
\end{aligned}
$$




$$
\begin{aligned}
& 1-\left(1-T_{\tilde{n}_{1}}\right)^{w_{1}}+1-\left(1-T_{\tilde{n}_{2}}\right)^{w_{2}} \\
& \left.-\left(1-\left(1-T_{\tilde{n}_{1}}\right)^{w_{1}}\right)\left(1-\left(1-T_{\tilde{n}_{2}}\right)^{w_{2}}\right), I_{\tilde{n}_{1}}^{w_{1}} I_{\tilde{n}_{2}}^{w_{2}}, F_{\tilde{n}_{1}}^{w_{1}} F_{\tilde{n}_{2}}^{w_{2}}\right\rangle .
\end{aligned}
$$

(2) When $n=k$, by applying Eq. (13), we get

$$
\begin{aligned}
\operatorname{TNWAA}\left(\tilde{n}_{1}, \tilde{n}_{2}, \ldots, \tilde{n}_{k}\right)= & \sum_{j=1}^{k} w_{j} \tilde{n}_{j} \\
= & \left\langle\left(\sum_{j=1}^{k} w_{j} a_{j 1}, \sum_{j=1}^{k} w_{j} a_{j 2}, \sum_{j=1}^{k} w_{j} a_{j 3}, \sum_{j=1}^{k} w_{j} a_{j 4}\right) ;\right. \\
& \left.1-\prod_{j=1}^{k}\left(1-T_{\tilde{n}_{j}}\right)^{w_{j}}, \prod_{j=1}^{k} I_{\tilde{n}_{j}}^{w_{j}}, \prod_{j=1}^{k} F_{\tilde{n}_{j}}^{w_{j}}\right\rangle
\end{aligned}
$$

(3) When $n=k+1$, by applying Eqs. (14) and (15), we can yield

$$
\begin{aligned}
\operatorname{TNWAA}\left(\tilde{n}_{1}, \tilde{n}_{2}, \ldots, \tilde{n}_{k+1}\right)= & \sum_{j=1}^{k+1} w_{j} \tilde{n}_{j} \\
= & \left\langle\left(\sum_{j=1}^{k+1} w_{j} a_{j 1}, \sum_{j=1}^{k+1} w_{j} a_{j 2}, \sum_{j=1}^{k+1} w_{j} a_{j 3}, \sum_{j=1}^{k+1} w_{j} a_{j 4}\right) ;\right. \\
& 1-\prod_{j=1}^{k}\left(1-T_{\tilde{n}_{j}}\right)^{w_{j}}+1-\left(1-T_{\tilde{n}_{k+1}}\right)^{w_{k+1}} \\
& -\left(1-\prod_{j=1}^{k}\left(1-T_{\tilde{n}_{j}}\right)^{w_{j}}\right) \\
& \left.\times\left(1-\left(1-T_{\tilde{n}_{k+1}}\right)^{w_{k+1}}\right), \prod_{j=1}^{k+1} I_{\tilde{n}_{j}}^{w_{j}}, \prod_{j=1}^{k+1} F_{\tilde{n}_{j}}^{w_{j}}\right\rangle \\
= & \left\langle\left(\sum_{j=1}^{k+1} w_{j} a_{j 1}, \sum_{j=1}^{k+1} w_{j} a_{j 2}, \sum_{j=1}^{k+1} w_{j} a_{j 3}, \sum_{j=1}^{k+1} w_{j} a_{j 4}\right) ;\right. \\
& \left.1-\prod_{j=1}^{k+1}\left(1-T_{\tilde{n}_{j}}^{w_{j}}\right), \prod_{j=1}^{k+1} I_{\tilde{n}_{j}}^{w_{j}}, \prod_{j=1}^{k+1} F_{\tilde{n}_{j}}^{w_{j}}\right\rangle
\end{aligned}
$$

Therefore, considering the above results, we have Eq. (13) for any $n$. This completes the proof. 
Obviously, the TNWAA operator satisfies the following properties:

(1) Idempotency: let $\tilde{n}_{j}(j=1,2, \ldots, n)$ be a collection of TNNs. If $\tilde{n}_{j}(j=$ $1,2, \ldots, n)$ is equal, i.e. $\tilde{n}_{j}=\tilde{n}$ for $j=1,2, \ldots, n$, then $\operatorname{TNWAA}\left(\tilde{n}_{1}, \tilde{n}_{2}, \ldots, \tilde{n}_{n}\right)=\tilde{n}$.

(2) Boundedness: let $\tilde{n}_{j}(j=1,2, \ldots, n)$ be a collection of TNNs and let $\tilde{n}^{-}=$ $\left\langle\left(\min _{j}\left(a_{j 1}\right), \min _{j}\left(a_{j 2}\right), \min _{j}\left(a_{j 3}\right), \min _{j}\left(a_{j 4}\right)\right) ; \min _{j}\left(T_{\tilde{n}_{j}}\right), \max _{j}\left(I_{\tilde{n}_{j}}\right), \max _{j}\left(F_{\tilde{n}_{j}}\right)\right\rangle$ and $\tilde{n}^{+}=\left\langle\left(\max _{j}\left(a_{j 1}\right), \max _{j}\left(a_{j 2}\right), \max _{j}\left(a_{j 3}\right), \max _{j}\left(a_{j 4}\right)\right) ; \max _{j}\left(T_{\tilde{n}_{j}}\right), \min _{j}\left(I_{\tilde{n}_{j}}\right), \min _{j}\left(F_{\tilde{n}_{j}}\right)\right\rangle$. Then $\tilde{n}^{-} \leqslant \operatorname{TNWAA}\left(\tilde{n}_{1} . \tilde{n}_{2}, \ldots, \tilde{n}_{n}\right) \leqslant \tilde{n}^{+}$.

(3) Monotonicity: let $\tilde{n}_{j}(j=1,2, \ldots, n)$ be a collection of TNNs. If $\tilde{n}_{j} \leqslant \tilde{n}_{j}^{*}$ for $j=1,2, \ldots, n$, then $\operatorname{TNWAA}\left(\tilde{n}_{1}, \tilde{n}_{2}, \ldots, \tilde{n}_{n}\right) \leqslant \operatorname{TNWAA}\left(\tilde{n}_{1}^{*}, \tilde{n}_{2}^{*}, \ldots, \tilde{n}_{n}^{*}\right)$.

Proof. (1) Since $\tilde{n}_{j}=\tilde{n}$ for $j=1,2, \ldots, n$, we have

$$
\begin{aligned}
\operatorname{TNWAA}\left(\tilde{n}_{1}, \tilde{n}_{2}, \ldots, \tilde{n}_{n}\right)= & \sum_{j=1}^{n} w_{j} \tilde{n}_{j} \\
= & \left\langle\left(\sum_{j=1}^{n} w_{j} a_{j 1}, \sum_{j=1}^{n} w_{j} a_{j 2}, \sum_{j=1}^{n} w_{j} a_{j 3}, \sum_{j=1}^{n} w_{j} a_{j 4}\right) ;\right. \\
& \left.1-\prod_{j=1}^{n}\left(1-T_{\tilde{n}_{j}}\right)^{w_{j}}, \prod_{j=1}^{n} I_{\tilde{n}_{j}}^{w_{j}}, \prod_{j=1}^{n} F_{\tilde{n}_{j}}^{w_{j}}\right\rangle \\
= & \left\langle\left(a_{1} \sum_{j=1}^{n} w_{j}, a_{2} \sum_{j=1}^{n} w_{j}, a_{3} \sum_{j=1}^{n} w_{j}, a_{4} \sum_{j=1}^{n} w_{j}\right) ;\right. \\
& \left.\times\left(1-\left(1-T_{\tilde{n}}\right)^{\sum_{j=1}^{n} w_{j}}\right), I_{\tilde{n}}^{\sum_{j=1}^{n} w_{j}}, F_{\tilde{n}}^{\sum_{j=1}^{n} w_{j}}\right\rangle \\
= & \left\langle\left(a_{1}, a_{2}, a_{3}, a_{4}\right) ; 1-\left(1-T_{\tilde{n}}\right), I_{\tilde{n}}, F_{\tilde{n}}\right\rangle \\
= & \tilde{n} .
\end{aligned}
$$

(2) Since the minimum TNN is $\tilde{n}^{-}$and the maximum TNN is $\tilde{n}^{+}$, there is $\tilde{n}^{-} \leqslant \tilde{n}_{j} \leqslant$ $\tilde{n}^{+}$. Thus, there is $\sum_{j=1}^{n} w_{j} \tilde{n}^{-} \leqslant \sum_{j=1}^{n} w_{j} \tilde{n}_{j} \leqslant \sum_{j=1}^{n} w_{j} \tilde{n}^{+}$. According to the above property (1), there is $\tilde{n}^{-} \leqslant \sum_{j=1}^{n} w_{j} \tilde{n}_{j} \leqslant \tilde{n}^{+}$, i.e. $\tilde{n}^{-} \leqslant T N W A A\left(\tilde{n}_{1}, \tilde{n}_{2}, \ldots, \tilde{n}_{n}\right) \leqslant \tilde{n}^{+}$.

(3) Since $\tilde{n}_{j} \leqslant \tilde{n}_{j}^{*}$ for $j=1,2, \ldots, n$, there is $\sum_{j=1}^{n} w_{j} \tilde{n}_{j} \leqslant \sum_{j=1}^{n} w_{j} \tilde{n}_{j}^{*}$, i.e. $\operatorname{TNWAA}\left(\tilde{n}_{1}, \tilde{n}_{2}, \ldots, \tilde{n}_{n}\right) \leqslant \operatorname{TNWAA}\left(\tilde{n}_{1}^{*}, \tilde{n}_{2}^{*}, \ldots, \tilde{n}_{n}^{*}\right)$.

Thus, we complete the proofs of these properties.

\subsection{Trapezoidal Neutrosophic Weighted Geometric Averaging Operator}

Definition 11. Let $\tilde{n}_{j}=\left\langle\left(a_{j 1}, a_{j 2}, a_{j 3}, a_{j 4}\right) ; T_{\tilde{n}_{j}}, I_{\tilde{n}_{j}}, F_{\tilde{n}_{j}}\right\rangle(j=1,2, \ldots, n)$ be a collection of TNNs, then a TNWGA operator is defined as follows:

$$
\operatorname{TNWGA}\left(\tilde{n}_{1}, \tilde{n}_{2}, \ldots, \tilde{n}_{n}\right)=\prod_{j=1}^{n} \tilde{n}_{j}^{w_{j}},
$$


where $w_{j}$ is the weight of $\tilde{n}_{j}(j=1,2, \ldots, n)$ such that $w_{j}>0$ and $\sum_{j=1}^{n} w_{j}=1$. Specially, when $w_{j}=1 / n$ for $j=1,2, \ldots, n$, the TNWGA operator reduces to the trapezoidal neutrosophic geometric averaging operator.

According to Definitions 7 and 11, we can introduce the following theorem.

Theorem 2. Let $\tilde{n}_{j}=\left\langle\left(a_{j 1}, a_{j 2}, a_{j 3}, a_{j 4}\right) ; T_{\tilde{n}_{j}}, I_{\tilde{n}_{j}}, F_{\tilde{n}_{j}}\right\rangle(j=1,2, \ldots, n)$ be a collection of TNNs, then according to Definitions 7 and 11, the following TNWGA operator is given by

$$
\begin{aligned}
\operatorname{TNWGA}\left(\tilde{n}_{1}, \tilde{n}_{2}, \ldots, \tilde{n}_{n}\right)= & \prod_{j=1}^{n} \tilde{n}_{j}^{w_{j}} \\
= & \left\langle\left(\prod_{j=1}^{n} a_{j 1}^{w_{j}}, \prod_{j=1}^{n} a_{j 2}^{w_{j}}, \prod_{j=1}^{n} a_{j 3}^{w_{j}}, \prod_{j=1}^{n} a_{j 4}^{w_{j}}\right) ;\right. \\
& \left.\prod_{j=1}^{n} T_{\tilde{n}_{j}}^{w_{j}}, 1-\prod_{j=1}^{n}\left(1-I_{\tilde{n}_{j}}\right)^{w_{j}}, 1-\prod_{j=1}^{n}\left(1-F_{\tilde{n}_{j}}\right)^{w_{j}}\right\rangle
\end{aligned}
$$

where $w_{j}$ is the weight of $\tilde{n}_{j}(j=1,2, \ldots, n)$ such that $w_{j}>0$ and $\sum_{j=1}^{n} w_{j}=1$.

By a similar proof manner of Theorem 1, we can prove Theorem 2, which is not repeated here.

Obviously, the TNWGA operator satisfies the following properties:

(1) Idempotency: let $\tilde{n}_{j}(j=1,2, \ldots, n)$ be a collection of TNNs. If $\tilde{n}_{j}(j=$ $1,2, \ldots, n)$ is equal, i.e. $\tilde{n}_{j}=\tilde{n}$ for $j=1,2, \ldots, n$, then $T N W G A\left(\tilde{n}_{1}, \tilde{n}_{2}, \ldots, \tilde{n}_{n}\right)=\tilde{n}$.

(2) Boundedness: let $\tilde{n}_{j}(j=1,2, \ldots, n)$ be a collection of TNNs and let $\tilde{n}^{-}=$ $\left\langle\left(\min _{j}\left(a_{j 1}\right), \min _{j}\left(a_{j 2}\right), \min _{j}\left(a_{j 3}\right), \min _{j}\left(a_{j 4}\right)\right) ; \min _{j}\left(T_{\tilde{n}_{j}}\right), \max _{j}\left(I_{\tilde{n}_{j}}\right), \max _{j}\left(F_{\tilde{n}_{j}}\right)\right\rangle$ and $\tilde{n}^{+}=\left\langle\left(\max _{j}\left(a_{j 1}\right), \max _{j}\left(a_{j 2}\right), \max _{j}\left(a_{j 3}\right), \max _{j}\left(a_{j 4}\right)\right) ; \max _{j}\left(T_{\tilde{n}_{j}}\right), \min _{j}\left(I_{\tilde{n}_{j}}\right), \min _{j}\left(F_{\tilde{n}_{j}}\right)\right\rangle$. Then $\tilde{n}^{-} \leqslant \operatorname{TNWGA}\left(\tilde{n}_{1}, \tilde{n}_{2}, \ldots, \tilde{n}_{n}\right) \leqslant \tilde{n}^{+}$.

(3) Monotonicity: let $\tilde{n}_{j}(j=1,2, \ldots, n)$ be a collection of TNNs. If $\tilde{n}_{j} \leqslant \tilde{n}_{j}^{*}$ for $j=1,2, \ldots, n$, then $\operatorname{TNWGA}\left(\tilde{n}_{1}, \tilde{n}_{2}, \ldots, \tilde{n}_{n}\right) \leqslant T N W G A\left(\tilde{n}_{1}^{*}, \tilde{n}_{2}^{*}, \ldots, \tilde{n}_{n}^{*}\right)$.

By a similar proof manner of the above properties, we can prove these properties (omitted).

\section{Decision Making Method with TNNs}

In this section, we apply the TNWAA and TNWGA operators and the score function to multiple attribute decision making problems under a TNN environment. For a multiple attribute decision making problem, assume that there are a set of alternatives $A=$ $\left\{A_{1}, A_{2}, \ldots, A_{m}\right\}$ based on a set of attributes $C=\left\{C_{1}, C_{2}, \ldots, C_{n}\right\}$. The weight vector 
Table 1

Linguistic values of TNNs for the linguistic term set.

\begin{tabular}{ll}
\hline Linguistic term & Linguistic value of TNNs \\
\hline Very poor & $\langle(0.1,0.1,0.1,0.1) ; 0.5,0.3,0.3\rangle$ \\
Poor & $\langle(0.2,0.3,0.4,0.5) ; 0.6,0.2,0.2\rangle$ \\
Fairly poor & $\langle(0.3,0.4,0.5,0.6) ; 0.7,0.1,0.1\rangle$ \\
Medium & $\langle(0.4,0.5,0.6,0.7) ; 0.8,0.0,0.1\rangle$ \\
Fairly good & $\langle(0.5,0.6,0.7,0.8) ; 0.8,0.1,0.1\rangle$ \\
Good & $\langle(0.7,0.8,0.9,1.0) ; 0.8,0.2,0.2\rangle$ \\
Very good & $\langle(1.0,1.0,1.0,1.0) ; 0.9,0.1,0.1\rangle$ \\
\hline
\end{tabular}

of the attributes is $W=\left(w_{1}, w_{2}, \ldots, w_{n}\right)^{T}$, which is given by the decision maker. Then, the decision maker can evaluate the alternatives on the attributes by the linguistic values of TNNs from the linguistic term set $L=\{$ Very poor, Poor, Fairly poor, Medium, Fairly good, Good, Very good\}, which are shown in Table 1. In the evaluation process, the decision maker can easily assign the linguistic values of TNNs to the attributes according to the linguistic terms, hence the evaluation information of the alternative $A_{i}$ on the attribute $C_{j}$ is represented by the form of a TNN $\tilde{n}_{i j}=\left\langle\left(a_{i j 1}, a_{i j 2}, a_{i j 3}, a_{i j 4}\right) ; T_{i j}, I_{i j}, F_{i j}\right\rangle$ $(i=1,2, \ldots, m ; j=1,2, \ldots, n)$. Thus, we can establish a trapezoidal neutrosophic decision matrix $D=\left(\tilde{n}_{i j}\right)_{m \times n}$.

Then, we apply the TNWAA or TNWGA operator and the score function to the multiple attribute decision making problems with trapezoidal neutrosophic information to rank the alternatives and to select the best one. The steps of the decision making process are described as follows:

Step 1: Utilize the TNWAA operator of Eq. (13) to obtain the collective overall number $\tilde{n}_{i}$ for $A_{i}(i=1,2, \ldots, m)$ with respect to the weight vector $W=\left(w_{1}, w_{2}, \ldots, w_{n}\right)^{T}$ for $C_{j}(j=1,2, \ldots, n)$ or the TNWGA operator of Eq. (18) to obtain the collective overall value $\tilde{n}_{i}$ for $A_{i}(i=1,2, \ldots, m)$ with respect to the weight vector $W=$ $\left(w_{1}, w_{2}, \ldots, w_{n}\right)^{T}$ for $C_{j}(j=1,2, \ldots, n)$.

Step 2: Calculate the score function $S\left(\tilde{n}_{i}\right)(i=1,2, \ldots, m)$ of the collective overall number $\tilde{n}_{i}(i=1,2, \ldots, m)$.

Step 3: Rank the alternatives according to the score values, and then select the best one. Step 4: End.

\section{An Illustrative Example}

In order to demonstrate the application of the proposed method, an example about the investment selection of a company is adapted from Ye (2014c). There is a company, which wants to invest a sum of money to an industry. A panel considers four alternatives: (1) $A_{1}$ is a car company; (2) $A_{2}$ is a food company; (3) $A_{3}$ is a computer company; (4) $A_{4}$ is an arms company. The evaluation on the alternatives is based on three attributes: (1) $C_{1}$ is the risk; (2) $C_{2}$ is the growth; (3) $C_{3}$ is the environmental impact. The weight vector of the three attributes is $W=(0.35,0.25,0.4)^{T}$. Then the four possible alternatives with respect 
to the above three attributes are evaluated by the expert or decision maker according to the linguistic values of TNNs for the linguistic term set in Table 1. Thus, we can establish the following trapezoidal neutrosophic decision matrix $D$ :

$$
\begin{aligned}
D= & {\left[\begin{array}{ll}
\langle(0.2,0.3,0.4,0.5) ; 0.6,0.2,0.2\rangle & \langle(0.3,0.4,0.5,0.6) ; 0.7,0.1,0.1\rangle \\
\langle(0.4,0.5,0.6,0.7) ; 0.8,0.0,0.1\rangle & \langle(0.5,0.6,0.7,0.8) ; 0.8,0.1,0.1\rangle \\
\langle(0.2,0.3,0.4,0.5) ; 0.6,0.2,0.2\rangle & \langle(0.3,0.4,0.5,0.6) ; 0.7,0.1,0.1\rangle \\
\langle(0.7,0.8,0.9,1.0) ; 0.8,0.2,0.2\rangle & \langle(0.5,0.6,0.7,0.8) ; 0.8,0.1,0.1\rangle \\
& \langle(0.2,0.3,0.4,0.5) ; 0.6,0.2,0.2\rangle \\
& \langle(0.3,0.4,0.5,0.6) ; 0.7,0.1,0.1\rangle \\
& \langle(0.3,0.4,0.5,0.6) ; 0.7,0.1,0.1\rangle \\
& \langle(0.3,0.4,0.5,0.6) ; 0.7,0.1,0.1\rangle
\end{array}\right] }
\end{aligned}
$$

Hence, the proposed method can be applied to this decision making problem according to the following computational process:

Step 1: Utilize the TNWAA operator of Eq. (13) to obtain the collective overall value $\tilde{n}_{i}$ for $A_{i}(i=1,2,3,4)$ as follows:

$$
\begin{aligned}
& \tilde{n}_{1}=\langle(0.2250,0.3250,0.4250,0.5250) ; 0.6278,0.1682,0.1682\rangle, \\
& \tilde{n}_{2}=\langle(0.3850,0.4850,0.5850,0.6850) ; 0.7648,0,0.1000\rangle, \\
& \tilde{n}_{3}=\langle(0.2650,0.3650,0.4650,0.5650) ; 0.6682,0.1275,0.1275\rangle, \\
& \tilde{n}_{4}=\langle(0.4900,0.5900,0.6900,0.7900) ; 0.7648,0.1275,0.1275\rangle .
\end{aligned}
$$

Step 2: Calculate the score values of $S\left(\tilde{n}_{i}\right)(i=1,2,3,4)$ of the collective overall value $\tilde{n}_{i}(i=1,2,3,4)$ by Eq. (11), we can obtain:

$$
S\left(\tilde{n}_{1}\right)=0.2864, \quad S\left(\tilde{n}_{2}\right)=0.4752, \quad S\left(\tilde{n}_{3}\right)=0.3338, \quad \text { and } \quad S\left(\tilde{n}_{4}\right)=0.5354 .
$$

Step 3: Ranking order of the four alternatives is $A_{4} \succ A_{2} \succ A_{3} \succ A_{1}$ according to the score values. Thus, the alternative $A_{4}$ is the best choice among the four alternatives.

On the other hand, we can also utilize the TNWGA operator to give the following computational procedure:

Step 1': By utilizing the TNWGA operator of Eq. (18) for $A_{i}(i=1,2,3,4)$, each collective overall value $\tilde{n}_{i}(i=1,2,3,4)$ is obtained as follows:

$$
\begin{aligned}
& \tilde{n}_{1}=\langle(0.2213,0.3224,0.4229,0.5233) ; 0.6236,0.1761,0.1761\rangle, \\
& \tilde{n}_{2}=\langle(0.3770,0.4786,0.5797,0.6805) ; 0.7584,0.0662,0.1000\rangle, \\
& \tilde{n}_{3}=\langle(0.2603,0.3617,0.4624,0.5629) ; 0.6632,0.1363,0.1363\rangle, \\
& \tilde{n}_{4}=\langle(0.4585,0.5642,0.6681,0.7710) ; 0.7584,0.1363,0.1363\rangle .
\end{aligned}
$$


Step 2': By using Eq. (11), we calculate the score values of $S\left(\tilde{n}_{i}\right)(i=1,2,3,4)$ of the collective overall value $\tilde{n}_{i}(i=1,2,3,4)$ as follows:

$$
S\left(\tilde{n}_{1}\right)=0.2820, \quad S\left(\tilde{n}_{2}\right)=0.4570, \quad S\left(\tilde{n}_{3}\right)=0.3282, \quad \text { and } \quad S\left(\tilde{n}_{4}\right)=0.5100 .
$$

Step 3': Hence, the ranking order of the four alternatives is $A_{4} \succ A_{2} \succ A_{3} \succ A_{1}$. Thus, the alternative $A_{4}$ is still the best choice among the four alternatives.

Obviously, above two kinds of ranking orders and the best alternative are identical and the same as Ye's results (2014c).

Compared with the relevant paper (Wang and Zhang, 2009) which proposed the intuitionistic trapezoidal fuzzy decision making approach, the decision making method in this paper uses the information of TNNs, whereas the decision making method in Wang and Zhang (2009) uses the information of ITFNs. As mentioned above, the TNN is a further generalization of the ITFN. So the decision making method proposed in this paper is more typical and more general in actual applications since the decision making method proposed in Wang and Zhang (2009) is a special case of the decision making method proposed in this paper. Furthermore, compared with the relevant papers (Ye, 2013; Chi and Liu, 2013; Ye, 2014a, 2014b, 2014c, 2014d; Liu et al., 2014; Zhang et al., 2014; Biswas et al., 2014; Zhang and Wu, 2014), the decision-making approach proposed in this paper can be used to solve decision making problems with triangular and trapezoidal neutrosophic information, whereas the decision-making methods in Ye (2013), Chi and Liu (2013), Ye (2014a, 2014b, 2014c, 2014d), Liu et al. (2014), Zhang et al. (2014), Biswas et al. (2014), Zhang and $\mathrm{Wu}$ (2014) are not suitable for the decision making problems in this paper because the domains of SVNSs and INSs are discrete sets, but not continuous sets in existing literature. Therefore, the method proposed in the paper is a further generalization of existing methods.

\section{Conclusions}

This paper proposed neutrosophic numbers and TNNs and the operational relations of TNNs as the extension of IFNs and ITFNs and introduced the score function of a TNN for comparing TNNs. Then we developed the TNWAA and TNWGA operators to aggregate TNNs and investigated their properties. Further, we established a decision making method based on the TNWAA or TNWGA operator and the score function to solve multiple attribute decision making problems with TNN information. Finally, an illustrative example was given to show the application of the developed decision making method. Since this paper extends the discrete domains of SVNSs and INSs to the continuous domain of TNNs, the developed decision making method includes much information and the fuzziness character in multiple attribute decision making problems with TNN information. In the future research, it is necessary to investigate the applications of these aggregation operators to the other domains such as pattern recognition and medical diagnosis.

Acknowledgement. This paper was supported by the National Natural Science Foundation of China (No. 71471172). 


\section{References}

Atanassov, K. (1986). Intuitionistic fuzzy sets. Fuzzy Sets and Systems, 20, 87-96.

Atanassov, K., Gargov, G. (1989). Interval valued intuitionistic fuzzy sets. Fuzzy Sets and Systems, 31, $343-349$.

Biswas, P., Pramanik, S., Giri, B.C. (2014). A new methodology for neutrosophic multi-attribute decisionmaking with unknown weight information. Neutrosophic Sets and Systems, 3, 42-50.

Broumi, S., Smarandache, F. (2014). Cosine similarity measure of interval valued neutrosophic sets. Neutrosophic Sets and Systems, 5, 15-20.

Broumi, S., Smarandache, F. (2015). New operations on interval neutrosophic sets. Journal of new theory, 1, 24-37.

Chi, P.P., Liu, P.D. (2013). An extended TOPSIS method for the multiple attribute decision making problems based on interval neutrosophic sets. Neutrosophic Sets and Systems, 1, 63-70.

Liu, P., Yu, X. (2013). Density aggregation operators based on the intuitionistic trapezoidal fuzzy numbers for multiple attribute decision making. Technological and Economic Development of Economy, 19(sup1), S454-S470

Liu, P.D., Chu, Y.C., Li, Y.W., Chen, Y.B. (2014). Some generalized neutrosophic number Hamacher aggregation operators and their application to group decision making. International Journal of Fuzzy Systems, 16(2), 242-255.

Meng, F., Chen, X. (2014). An approach to interval-valued hesitant fuzzy multi-attribute decision making with incomplete weight information based on hybrid Shapley operators. Informatica, 25(4), 617-642.

Smarandache, F. (1999). A Unifying Field in Logics. Neutrosophy: Neutrosophic Probability, Set and Logic. American Research Press, Rehoboth.

Turksen, I. (1986). Interval valued fuzzy sets based on normal forms. Fuzzy Sets and Systems, 20, 191-210.

Wan, S., Dong, J. (2014). Multi-attribute group decision making with trapezoidal intuitionistic fuzzy numbers and application to stock selection. Informatica, 25(4), 663-697.

Wang, W., Liu, X. (2014). Some hesitant fuzzy geometric operators and their application to multiple attribute group decision making. Technological and Economic Development of Economy, 20(3), 371-390.

Wang, J.Q., Zhang, Z. (2009) Aggregation operators on intuitionistic trapezoidal fuzzy number and its application to multi-criteria decision making problems. Systems Engineering and Electronics, 20(2), 321-326.

Wang, H., Smarandache, F., Zhang, Y.Q., Sunderraman, R. (2005). Interval Neutrosophic Sets and Logic: Theory and Applications in Computing. Hexis, Phoenix, AZ.

Wang, H., Smarandache, F., Zhang, Y.Q., Sunderraman, R. (2010). Single valued neutrosophic sets. Multispace and Multistructure, 4, 410-413.

Ye, J. (2013). Multicriteria decision-making method using the correlation coefficient under single-valued neutrosophic environment. International Journal of General Systems, 42(4), 386-394.

Ye, J. (2014a). Similarity measures between interval neutrosophic sets and their applications in multicriteria decision-making. Journal of Intelligent and Fuzzy Systems, 26, 165-172.

Ye, J. (2014b). Single valued neutrosophic cross-entropy for multicriteria decision making problems. Applied Mathematical Modelling, 38, 1170-1175.

Ye, J. (2014c). A multicriteria decision-making method using aggregation operators for simplified neutrosophic sets. Journal of Intelligent and Fuzzy Systems, 26, 2459-2466.

Ye, J. (2014d). Vector similarity measures of simplified neutrosophic sets and their application in multicriteria decision making. International Journal of Fuzzy Systems, 16(2), 204-211.

Ye, J. (2014e). Some aggregation operators of interval neutrosophic linguistic numbers for multiple attribute decision making. Journal of Intelligent and Fuzzy Systems, 27(5), 2231-2241.

Zadeh, L.A. (1965). Fuzzy sets. Information and Control, 8(5), 338-353.

Zhang, Z., Wu, C. (2014). A novel method for single valued neutrosophic multi-criteria decision making with incomplete weight information. Neutrosophic Sets and Systems, 4, 35-49.

Zhang, H.Y., Wang, J.Q., Chen, X.H. (2014). Interval neutrosophic sets and their application in multicriteria decision making problems. The Scientific World Journal, 2014, Article ID 645953, 15 pp.

Zhou, W., He, J.M. (2014). Interval-valued intuitionistic fuzzy ordered precise weighted aggregation operator and its application in group decision making. Technological and Economic Development of Economy, 20(4), $648-672$. 
J. Ye graduated and received his MS degree in automation and robotics from the Technical University of Koszalin, Poland in 1997. From February 2012 to August 2012, he was a visiting scholar in the School of Engineering of Southern Polytechnic State University in USA. Now, he is a professor in the Department of Electrical and Information Engineering, Shaoxing University, China. He has more than 30 years of experience in teaching and research. His research interests include soft computing, fuzzy decision-making, pattern recognitions, fault diagnosis, robotics, and intelligent control. He has published more than one hundred papers in journals. He has written a few books related to his research work. He has finished a few projects sponsored by government of China. 